International Journal of Advanced Astronomy, 8(1) (2020) 27-28
International Journal of Advanced Astronomy
SPC
Website: $w$ ww. sciencepubco.com/index.php/IJAA
Research paper

\title{
Dark comets: the cosmic catastrophic threat to earth
}

\author{
M. Khalil ${ }^{1 *}$, M. Said ${ }^{2}$, H. Osman ${ }^{1}$, N. Younis ${ }^{2}$, N. Khaled ${ }^{1}$, Y. Amr ${ }^{1}$, N.A. Mohamed ${ }^{1}$, A. Ibrahim ${ }^{1}$ \\ ${ }^{1}$ Faculty of Engineering, October University for Modern Sciences and Arts (MSA), Giza, Egypt \\ ${ }^{2}$ Department of English and American studies, University of Vienna, Austria \\ *Corresponding author E-mail: mkibrahim@msa.eun.eg
}

\begin{abstract}
Dark Comets are catastrophic hazardous objects that could hit Earth without warning. There are thousands of such unseen comets buzzing around. Very few of which are known. An overview of the dark comets deadly threat is presented in this paper. Moreover, detection techniques of these invisible objects are discussed.
\end{abstract}

Keywords: Dark Comets; Unseen Interstellar Objects; Comets Detection.

\section{Introduction}

Comets are icy interstellar bodies in space that move at high speed releasing gases and dust [2], [5]. A comet does not emit light but it reflects sunlight due to its icy body. Dark comets are usually unseen potential threats to Earth. A dark comet is very difficult to spot as it doesn't glow and it only reflects a small fraction of light. The main reason of the low albedo is that there is little or no ice on the outer surface of the comet. On the other hand, the surface of the dark comet contains minerals like iron sulfides and carbon-based compounds [4], [5]. The rest of the paper is organized as follows. In Section 2, a discussion about the hazard of dark comets while section 3 is devoted to discuss the detection techniques of dark comets.

\section{Are dark comets the biggest hazard to earth?}

Fast-moving, invisible large objects like dark comets could strike our planet Earth and cause devastating effects on life on Earth. Thousands of such dark comets are unseen in our Solar System, of which only very few are known [1]. When a comet comes closer to the sun, it heats up and the ice on the surface of the nucleus become gas causing the development of a long tail and the comet can be easily observable from sky surveys before too much time has passed. The comet's tail can stretch out up to hundreds of millions of kilometers in length. For example, Comet Hyakutake's tail is more than 500 million $\mathrm{km}$ long. The tail of the dark comet is unseen and that makes everything invisible, and the comet cannot be spotted. Dark comets are invisible and may be spotted moving towards Earth just a few days before they come closer. In other words, a dark comet is considered as a potential threat to life on Earth if it could escape from early detection as it does not glow. In 1983 the comet IRAS-Araki-Alcock passed by the Earth at a distance of 5000,000 km which is the closest distance reached by a comet during the previous 200 years. The comet was observed just about two weeks before it came close to Earth [4], [5]. So, these dark comets come out of the dark and may hit Earth with no warning.

\section{Detection of dark comets}

Dark comets could be detected by the heat they emit. NASA launched an orbiting infrared telescope called the Wide-field Infrared Survey Explorer (WISE) on December 14, 2009 [3] to create infrared images of the sky. WISE mission is to discover objects including dark comets by sensing their heat in the form of infrared light [3]. Nevertheless, scientists observe dark comets near Earth using radar [3]. For example, scientists used the Goldstone Solar System Radar in USA to track the dark comet P/2016 BA14. An interesting mathematical model implemented in [3], [4] proposed that there are many invisible dark comets near Earth which are undetectable with current near-Earth object (NEO) search programs [5].

\section{Conclusion and recommendations}

This paper highlights the importance of studying the dark comets which are considered as the most dangerous threat to life on earth as such unseen comets could hit earth without warning. Detection techniques of such unseen comets are presented briefly in this work. From our point of view, it is very significant to implement new computational models to detect and track unseen comets. We believe that machine learning models should be developed in order to study and predict the behavior of the unseen comets. 


\section{References}

[1] Khalil, M., Said, M., Osman, H., Ahmed, B., Ahmed, D., Younis, N., Maher, B., Osama, M. and Ashmawy, M., Big data in astronomy: from evolution to revolution, International Journal of Advanced Astronomy, 7 (1) (2019) 11-14. https://doi.org/10.14419/ijaa.v7i1.18029.

[2] Napier, W.M., Wickramasinghe, J.T. and Wickramasinghe, N.C., 2004. Extreme albedo comets and the impact hazard. Monthly Notices of the Royal Astronomical Society, 355(1), pp.191-195.Peplow, M. Unseen comets may raise impact risk for Earth. Nature (2004). https://doi.org/10.1111/j.13652966.2004.08309.x.

[3] Peplow, M. Unseen comets may raise impact risk for Earth. Nature (2004). https://doi.org/10.1038/news041018-3.

[4] Astronomy now: Dark comet's flyby of Earth observed with radar and infrared (2016). https://astronomynow.com/2016/03/25/dark-comets-flybyof-earth-observed-with-radar-and-infrared/

[5] Homeland Security News wire: New threat to Earth: "Dark" comets (2009). http://www.homelandsecuritynewswire.com/new-threat-earth-dark-comets. 